\title{
Tumor de krukenberg metastático sem diagnóstico prévio de tumor primário: Relato de caso
}

\author{
Tumor of metastatic krukenberg without prior diagnosis of primary tumor: case report
}

Roberta Maria Rola Albergaria ${ }^{\dagger *}$, Júlia Pereira Alvim, Lucas Oliveira Salles ${ }^{\dagger}$, Maria Júlia Campos Guerra ${ }^{\dagger}$, Lucineide Martins de Oliveira Maia ${ }^{\ddagger}$ Aline Trovão Queiroz

Como citar esse artigo. Albergaria, RMR; Alvim, JP; Salles, LO; Guerra, MJC; Maia, LMO; Queiroz, AT Tumor de krukenberg metastático sem diagnóstico prévio de tumo primário: Relato de caso. Revista de Saúde. 2018 Jul./Dez.; 09 (2): 31-36.

\section{Resumo}

O tumor de Krukenberg (TK) é uma metástase neoplásica ovariana incomum, representa 1-2\% dos cânceres incidentes no ovário, nos quais o estômago é a localização primária mais habitual. O objetivo do estudo foi relatar um caso de uma paciente de 42 anos, com TK metastático diagnosticado após laparotomia de emergência, relacionado a uma neoplasia gástrica sem diagnóstico prévio. A média de idade encontrada em pacientes com TK é 45 anos, sendo que a maioria dos casos relatados trata-se de TK bilateral. Os sintomas podem ser inespecíficos, com diagnósticos incidentais intraopertórios, ou relacionados ao envolvimento ovarianos, com dor e distensão abdominal e irregularidade menstrual. Comumente os pacientes podem ser assintomáticos, tendo manifestações clínicas somente muito tardiamente. A expressão de receptores de hormônios sexuais no TK ainda é indeterminada. Em relação ao prognóstico, a metastasectomia promove uma maior sobrevida aos pacientes, enquanto a cirurgia citorredutora revelou efeitos benéficos em relação a sobrevida, porém pode associar-se ao aumento da morbimortalidade e recorrências. Ainda não há consenso definido na literatura sobre o tratamento ideal, embora haja avanços nos estudos. O TK é um diagnóstico raro, acomente mais mulheres na prémenopausa e tem um prognóstico ruim. A primeira recomendação para melhor sobrevida da paciente é a ressecção da lesão metastásica, seguida de terapia sistêmica paliativa.

Palavras-chave: Tumor de Krukenberg; Metástase Neoplásica; Neoplasias Gástricas; Neoplasias Ovarianas.

\begin{abstract}
Krukenberg's tumor (TK) is an unusual neoplastic ovarian metastasis, representing 1-2\% of cancers incident on the ovary, in which the stomach is the most common primary site. The objective of this study was to report a case of a 42 -yearold woman with metastatic TK diagnosed after emergency laparotomy, related to a gastric neoplasia without previous diagnosis. The mean age of patients with TK is 45 years, and the majority of reported cases are bilateral TK. Symptoms may be nonspecific, with incidental inoperative diagnoses, or ovarian involvement related, such as abdominal pain and distention and menstrual irregularity. Commonly there are asymptomatic patients, only having clinical manifestations very late. The expression of sex hormone receptors in TK is still indeterminate. About the prognostic, the metastasectomy promotes a greater survival to the patients, whereas the cytoreductive surgery revealed beneficial effects in relation to the survival, but it can be associated with the increase of morbimortality and recurrences. There is still no definite conguration in the literature on the ideal treatment, although there are advances in the studies. TK is a rare diagnosis, it is prevalent in premenopausal women and it has a bad prognosis. The first recommendation for better patient survival is the resection of the metastatic lesion, followed by systemic palliative therapy.
\end{abstract}

Keywords: Krukenberg Tumor; Neoplasm metastasis; Stomach Neoplasm; Ovarian Neoplasms.

\section{Introdução}

O Tumor de Krukenberg (TK) é um tumor de ovário metastático incomum, no qual o estômago é a localização primária mais frequente, seguida do cólon, apêndice e mama, respectivamente ${ }^{1}$. Representa 1-2\% dos cânceres incidentes no ovário ${ }^{1}$. Sua ocorrência unilateral não é usual ${ }^{2}$ e raramente o tumor primário apresenta grandes dimensões ${ }^{3}$. Representa $30-40 \%$ dos cânceres metastáticos de ovário ${ }^{4}$. Trata-se de um adenocarcinoma que afeta mulheres jovens, na grande maioria $(80 \%)$ das vezes acomete os ovários bilateralmente e são sólidos ${ }^{4}$. Pode ocorrer também, após resecção cirúrgica de câncer gástrico, com incidência entre $0,3 \%$ a $6,7 \%{ }^{4}$.

O TK foi descrito pela primeira vez em 1896

Afiliação dos autores: † Discente do Curso de Graduação em Medicina da Universidade de Vassouras, Vassouras, Rio de Janeiro, Brasil.

\$ Docente do Curso de Graduação em Medicina da Universidade de Vassouras, Vassouras, Rio de Janeiro, Brasil.

* Email de correpondência: roberta.albergaria@outlook.com 
por Friederich Krukenberg, quando estudava 6 casos de tumores ovarianos atípicos ${ }^{1}$. Histologicamente é definido como um carcinoma ovariano que contém células neoplásicas em anel de sinete cheias de mucina, sendo que aproximadamente 5\% de todos os carcinomas metastáticos dos ovários são adenocarcinoma ${ }^{5}$.

São descritas quatro possíveis vias de disseminação para a ocorrência da infiltração ovariana das células tumorais primárias: por contiguidade, via peritoneal, via linfática retrógrada (as células neoplásicas passariam pelos gânglios coronário-estomatais e pré-aórticos, mais tarde para a porção posterior e lombo-aórtica, e daí para o ovário - é a teoria mais aceita) e a disseminação via hematogênica (teoria de Virchow) ${ }^{1}$.

Alguns autores sugeriram que o aumento da vascularização dos ovários férteis ou a cicatrização ovariana após ovulação recente seria uma causa do aumento do número de TK em mulheres na prémenopausa ${ }^{6}$.

Otumor primário mais comumé oadenocarcinoma gástrico ${ }^{7}$. Estudos recentes indicam que o câncer gástrico é um dos cânceres mais comuns em todo o mundo, e a incidência é particularmente alta em países asiáticos, incluindo a China ${ }^{8}$.

Avanços significativos têm surgido para compreender a biologia molecular de muitos cânceres. No entanto, o mecanismo envolvido na heterogeneidade do câncer gástrico ainda não foi claramente estabelecido. Além disso, os fatores prognósticos e diretrizes para tratamento de pacientes diagnosticados com TK de origem gástrica ainda não são totalmente conclusivos ${ }^{4}$.

Metástase e recorrência são as principais causas de mau prognóstico no câncer gástrico. No caso das mulheres, a metástase ovariana inclui ressecção curativa do câncer gástrico ${ }^{8}$. Em relação à classificação do tumor, entende-se por tumor sincrônico, aquele segundo tumor primário diagnosticado com intervalo de até 6 meses em pacientes que já apresentavam um primeiro tumor primário e metacrônico quando o segundo tumor primário é diagnosticado com intervalo superior a 6 meses ${ }^{9}$.

A estratégia de tratamento ideal para os TK do câncer gástrico até hoje ainda não foi claramente estabelecida. Embora a quimioterapia sistêmica possa proporcionar alívio paliativo e sobrevida prolongada em pacientes com metástase ovariana, a eficiência e o tempo de sobrevida permanecem sem importância significativa. Vários estudos têm explorado a utilidade da metastasectomia para os TK em pacientes com câncer gástrico. Entretanto, o papel da metastasectomia ovariana ainda está em debate e provavelmente beneficiará apenas um subgrupo específico de pacientes ${ }^{8}$.

O presente artigo objetiva relatar um caso de TK metastático confirmado após laparotomia de emergência em mulher de 42 anos, associado à um câncer gástrico não diagnosticado previamente

\section{Relato de caso}

Paciente do sexo feminino, 42 anos, refere história de emagrecimento nos últimos meses, que a princípio foi relacionado ao fato de estar em dieta associada a exercício físico. Emagreceu $12 \mathrm{~kg}$ em aproximadamente 5 meses. Quando começou a emagrecer, notou que na fossa ilíaca esquerda havia um abaulamento, porém não deu importância. Nos últimos meses, percebeu abdome globoso, com possível suspeita de gravidez, descartada pelo exame beta HCG (gonadotrofina coriônica humana). Procurou o Hospital Universitário de Vassouras (HUV) referindo muita dor (não especificada), distensão abdominal e há 2 dias observou parada de eliminação de gases e fezes. Paciente apresentava-se emagrecida e com abdome ascítico. Foi realizada rotina de abdome agudo evidenciando distensão de alças e sem presença de gás no reto. Ao toque retal, não apresentava fezes na ampola, exame de sangue com leucograma normal. Diante da gravidade do quadro e suspeitando de abdome agudo obstrutivo, a paciente foi submetida a uma laparotomia exploratória (Figura1). Durante o procedimento, verificou-se a presença de grande quantidade de líquido ascítico (aproximadamente 5 litros), o qual foi aspirado. A incisão foi feita infra umbilical pois até então, não havia sido discutida a possibilidade de uma obstrução a um nível mais baixo. Notou-se o ovário esquerdo bastante aumentado, com forma irregular, comprimindo o sigmoide, evidenciando a causa da obstrução. Ao fazer o inventário da cavidade, percebeu-se o estômago endurecido e, dessa forma, a incisão foi ampliada até o nível xifopubiano, evidenciando o estômago como uma linite plástica, tomado de lesão tumoral, impossibilitando qualquer intervenção (Figura 2). Foi realizada ooforectomia esquerda (Figura 3) e coleta de líquido ascítico para a citologia (Figura 4). Optou-se em fazer uma jejunostomia para a paciente se alimentar, pois o estômago estava repleto de lesão tumoral e a paciente apresentava-se consumida. Além disso, foi feita uma colostomia em alça, pois a presença do abdômen agudo com sinal de obstrução devido à metástase ao redor, aumentava o risco de o quadro evoluir para uma obstrução total. Após análise histopatológica, confirmou-se a presença de adenocarcinoma no ovário, carcinoma no líquido peritoneal e adenocarcinoma no estômago.

\section{Discussão}

Mulheres com TK tendem a ser incomumente jovens para pacientes com carcinoma metastático, que geralmente vai ocorrer na quinta década de suas vidas, com uma média idade de 45 anos $^{3}$.

Até $30 \%$ das metástases ovarianas surgem de origem colorretal, mas o adenocarcinoma gástrico é a 
Figura 1. Laparotomia exploratória realizada por conta da suspeita do quadro de abdome agudo obstrutivo da paciente, a fim de detectar a possível causa da sintomatologia.

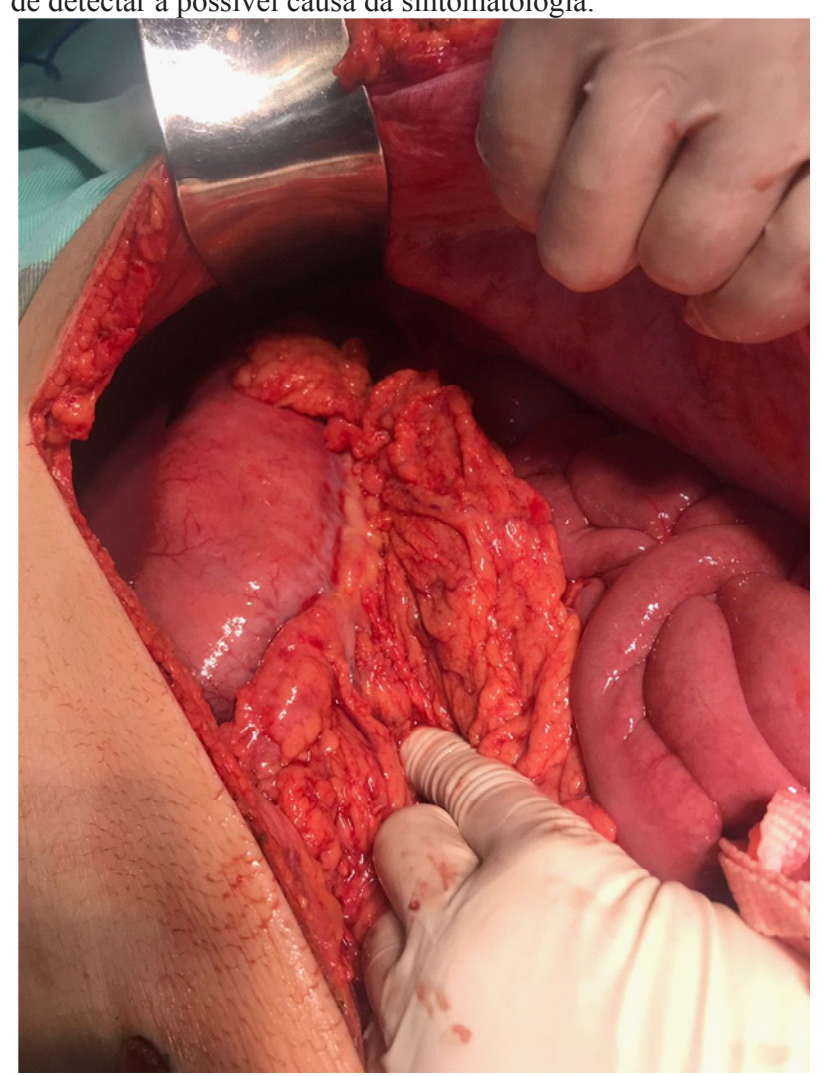

Figura 2. Tumor gástrico primário, o qual teve sua origem no estômago, que apresentou metástase no ovário esquerdo.

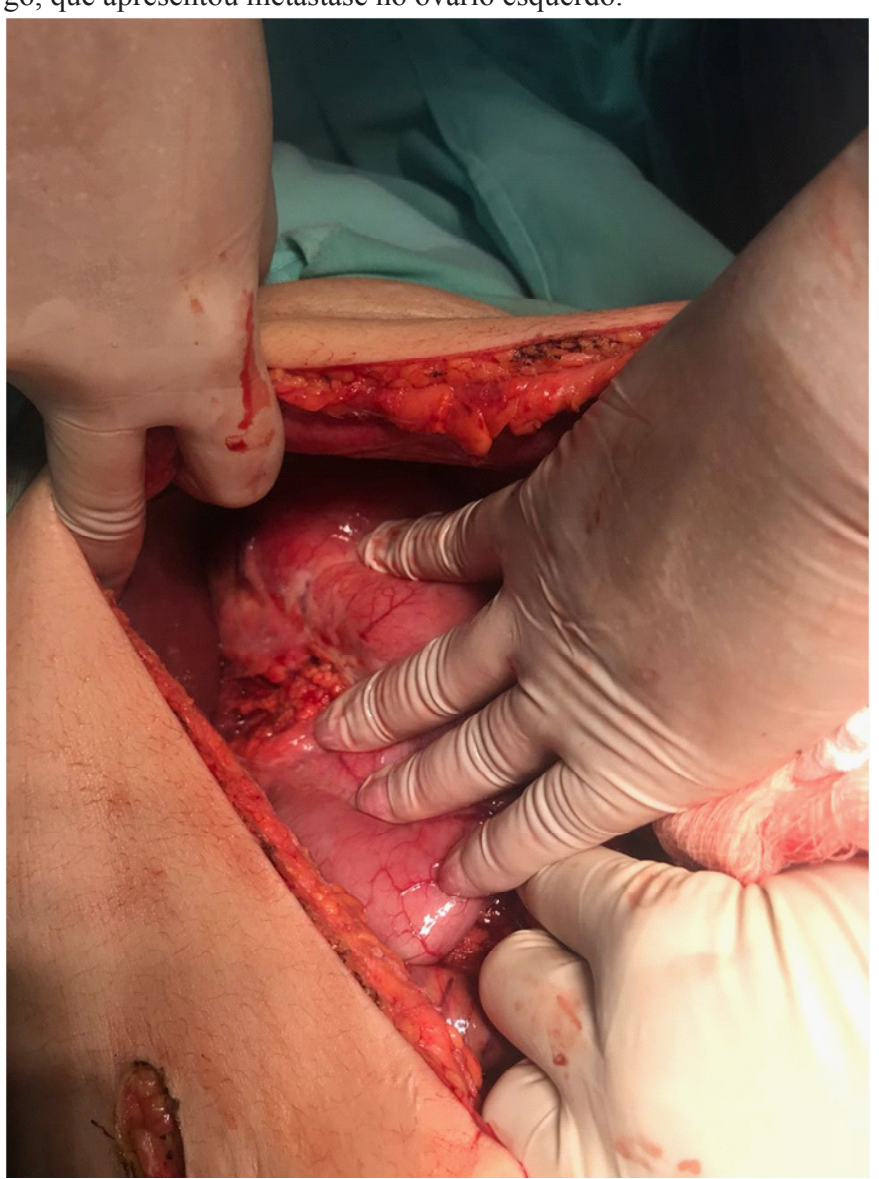




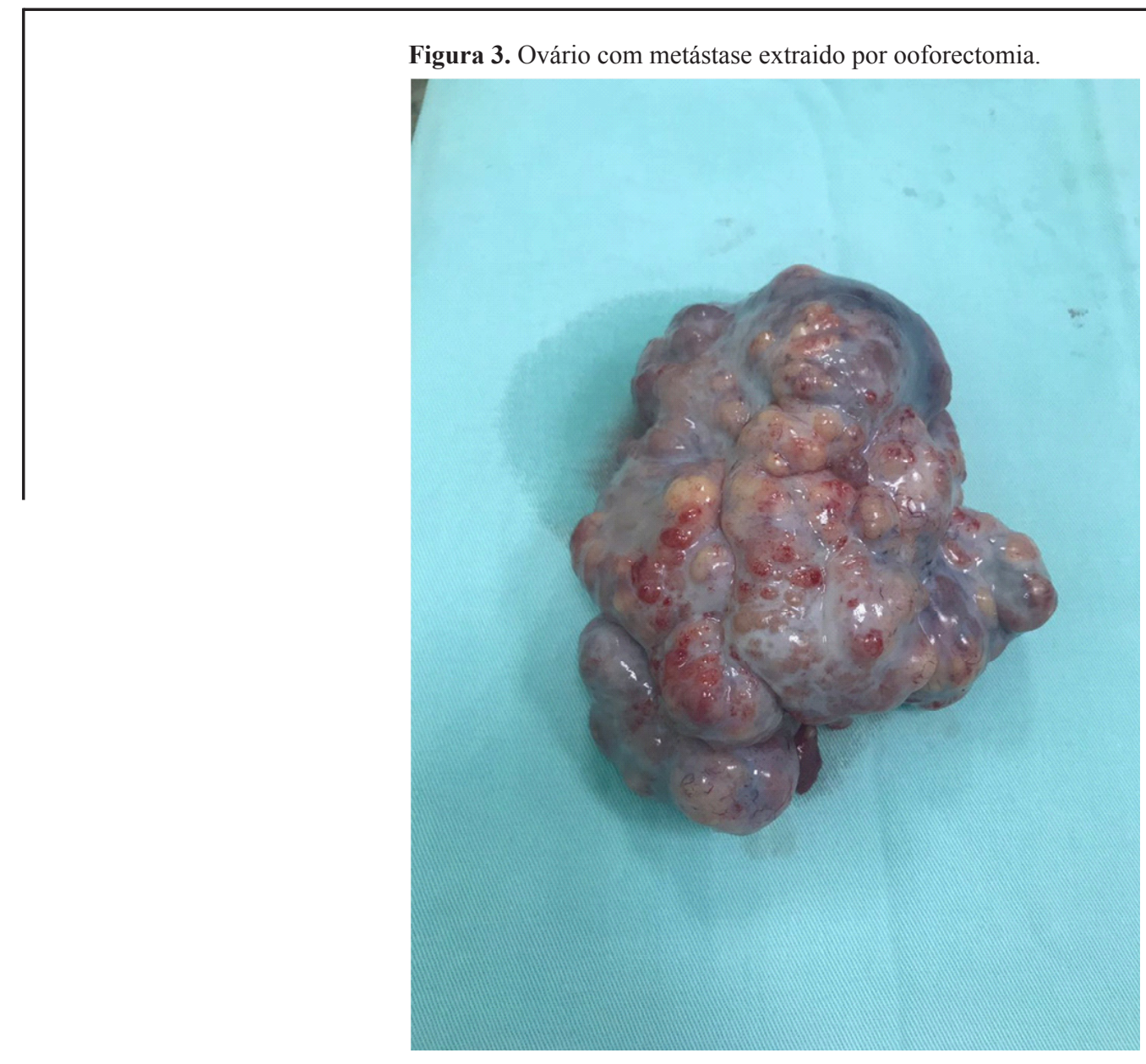

Figura 4. Líquido ascitico aspirado.

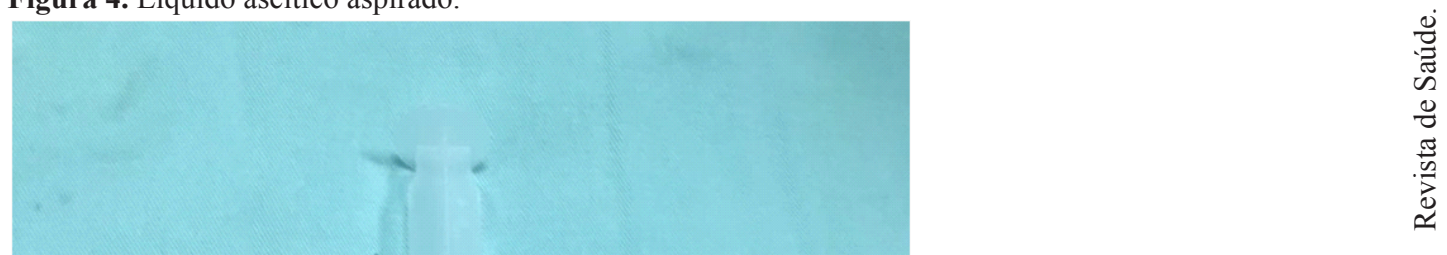


malignidade primária mais comum ${ }^{7}$, o que foi observado no caso relatado. Porém, a literatura recente tem descrito um aumento na incidência de tumores originados do cólon5.

Em comparação com tumores de ovário primários, o prognóstico dos pacientes com TK é sombrio principalmente quando a metástase está confinada aos ovários5. No caso em questão, a metástase estava confinada apenas em um ovário, mas a literatura refere que o acometimento bilateral é mais frequente ${ }^{8}$.

Os sintomas do TK são inespecíficos e em $30 \%$ dos casos pode ser a primeira manifestação do tumor primário, mas outras vezes são descobertas incidentalmente intraoperatório ${ }^{1}$, como descrito neste caso clínico. Em outros casos, o câncer gástrico pode ser pequeno e não ser detectado durante vários anos após ooforectomia ${ }^{10}$, ou ainda, nunca ser detectado ${ }^{2}$.

Em geral, eles constituem um dilema para a realização de diagnóstico, especialmente quando é apresentado como uma manifestação inicial sem que o tumor primário tenha sido descoberto ${ }^{1}$. No presente caso não havia um diagnóstico prévio de tumor gástrico primário, o qual suspeitado no momento da cirurgia, após ter sido observado uma massa tumoral extensa em ovário esquerdo.

Os sintomas geralmente estão relacionados ao envolvimento ovariano, os mais comuns são dor abdominal e distensão (principalmente quando bilateral e grandes massas ovarianas) ${ }^{3}$, mas algumas mulheres podem apresentar irregularidade menstrual ${ }^{10}$. Os demais pacientes têm sintomas gastrintestinais não específicos ou são assintomáticos ${ }^{3}$. Em geral, as manifestações clínicas são reveladas muito tardiamente, e as primeiras manifestações podem ser ascite, como no presente caso, ou carcinomatose peritoneal, que não ocorreu no caso descrito ${ }^{6}$.

A análise de fatores prognósticos pode facilitar a identificação de pacientes que teriam maior beneficio com o tratamento. Metastasectomia, carcinomatose peritoneal e expressão de receptores de estrogênio e progesterona - ER- $\beta$ e PR, respectivamente, foram considerados preditores independentes por alguns autores8. Esses, também afirmam que os TK são muitas vezes associados a diferentes graus de metástase peritoneal, que frequentemente induz a ascite intestinal, obstrução ou hidronefrose, podendo comprometer seriamente a qualidade de vida do paciente. Corroborando à literatura, a paciente em questão apresentou-se com queixa de obstrução intestinal, além da ascite moderada.

Alguns estudos relataram uma correlação entre a expressão de receptores de hormônios sexuais e a incidência e progressão do câncer gástrico, assim, tem sido sugerido que os hormônios sexuais desempenham um papel crítico em pacientes do sexo feminino com câncer gástrico. Recentemente, estudos sugeriram que uma maior exposição aos efeitos do estrogênio pode diminuir potencialmente o risco de câncer gástrico. Entretanto, a presença e o papel dos receptores de hormônios sexuais no TK ainda é indeterminada ${ }^{8}$.

Em relação aos fatores prognósticos e resultados da cirurgia para os TK, muitos estudos foram realizados no passado, verificou-se que a metastasectomia, ou a remoção cirúrgica de um ou ambos os ovários envolvidos promoviam uma melhor sobrevida do paciente $^{11}$, de modo que, além do aumento da sobrevida também gera alívio dos sintomas apresentados pela paciente. Além desses pontos positivos é importante salientar que a metastasectomia trata-se de um procedimento cirúrgico relativamente seguro e que requer curta internação hospitalar. O tempo médio entre a descoberta do TK e a metastasectomia é de 15 dias $^{8}$, mas no caso em questão foi concomitante, uma vez que a paciente submeteuse à laparotomia exploratória por sua queixa incial se tratar de abdome obstrutivo. A cirurgia citorredutora que incluía a remoção do TK também revelou ter efeitos benéficos, com $7 \%$ de sobrevida em 5 anos. No entanto, cirurgia citorredutora extensa poderia ter associação significativa com aumento da morbidade e mortalidade5, além de apresentar taxa de recorrência regional ou metastática significativa, apesar de alançar reesecção completa inicialmente ${ }^{7}$. Na paciente relatada optou-se por metastasectomia do ovário acometido, não remoção do tumor gástrico e posterior tratamento quimioterápico.

Em relação à sobrevida dos pacientes, em estudo realizado na China, no qual foram acompanhados casos de TK metastático de câncer gástrico durante 10 anos $^{8}$, os autores afirmam que a média de sobrevida de pacientes com tumores ovarianos metastático sincrônicos foi de 15,6 meses, sendo que quando feita a metatasectomia nesses pacientes a sobrevida subiu para 19 meses, mas quando não feita foi de 11,8 meses. Para as pacientes com tumores ovarianos metastático metacrônicos, a média de sobrevida foi de 21,7 meses, sendo que quando feita a metatasectomia nesses pacientes a sobrevida subiu para 24,6 meses, mas quando não feita foi de 14,3 meses. Observa-se que em ambos os casos a realização da metastasectomia possibilitou uma maior sobrevida, embora no caso dos tumores metacrônicos tenha sido maior do que a dos turmores sincrônicos ${ }^{8}$. No caso relatado, a paciente apresentou metástase ovariana síncrona e, objetivando uma maior sobrevida da mesma, optou-se por realizar a metastasectomia apenas do ovário acometido, já que o outro encontrava-se hígido.

\section{Conclusão}

Apesar de inúmeros avanços nos estudos sobre o melhor tratamento a ser instituído, não existe na literatura um consenso definido, e não há ainda um tratamento 
considerado ideal. A metastasectomia ovariana associada à quimioterapia têm sido preconizadas atualmente.

Conclui-se que o TK é uma entidade rara, acomete em geral mulheres na pré-menopausa e possui um prognóstico ruim logo ao seu diagnóstico. Ainda assim, a ressecção da lesão metastática é a primeira recomendação que pode melhorar a sobrevida da paciente, seguida de terapia sistêmica paliativa.

\section{Referências}

1. Lien TT, Iglesias OS, Carvajal LF. Tumor de Krukenberg. Rev Cubana de Obstetricia y Ginecología. 2014; 40 (1): 96-101.

2. Nandini G, Umadevi K, Padma K, Uday R. Krukenberg Tumor in Adolescent: A Rare Occurrence. Journal of South Asian Federation of Obstetrics and Gynaecology. 2014; 6 (3): 202-204

3. Al-Agha OM, Nicastri AD. An in-depth look at Krukenberg tumor: an overview. Rev Archives of pathology \& laboratory medicine. 2006; 130 (11): 1725-1730.

4. Durán DH, Mitjans OD, Capote R, Lorente RR, Jones DC. Tumor de Krukenberg. Rev Cubana de Obstetricia y Ginecología. 2011; 37 (3): 414419.

5. Nirmal AK, Nirmal K, Kumar S, Jha J. Krukenberg Tumour of the Ovary in young Female: A Case Report. Int J Curr Res Med Sci. 2016; 2 (10): 60-

6. Cho JH, Lim JY, Choi AR, Choi SM, Kim JW, Choi SH, et al. Comparison of surgery plus chemotherapy and palliative chemotherapy alone for advanced gastric cancer with Krukenberg tumor. Cancer research and treatment: official journal of Korean Cancer Association. 2015; 47 (4): 697.

7. Lam D, Ong E. The role of surgical excision for the Krukenberg tumour: A case report. International journal of surgery case report. 2017; 38: 185-188.

8. Yu P, Huang L, Cheng G, Yang L, Dai G, Ying J, Du Y. Treatment strategy and prognostic factors for Krukenberg tumors of gastric origin: report of a 10-year single-center experience from China. Rev Oncotarget. 2017; 8 (47): 82558 .

9. Brito Filho, AP. Estudo de prevalência de lesões sincrônicas e metacrônicas da mucosa do trato aerodigestivo superior: análise retrospectiva [dissertação]. Recife: Universidade Federal de Pernanbuco - UFPE; 2010.

10. Seow-En I, Hwarng G, Tan GHC, Ho LML, Teo MCC. Palliative surgery for Krukenberg tumors-12-year experience and review of the literature. World journal of clinical oncology. 2018; 9 (1): 13

11. Lu LC, Shao YY, Hsu CH, HSU C, Cheng WF, Lin YL, et al. Metastasectomy of Krukenberg tumors may be associated with survival benefits in patients with metastatic gastric cancer. Rev Anticancer research. 2012; 32 (8): 3397-3401. 